\title{
Ampliação das Possibilidades de Gamificação no Moodle
}

\author{
Eduardo Barrére, Marluce Aparecida Vitor, Miguel Alvim de Almeida \\ Departamento de Ciência da Computação - Universidade Federal de Juiz de Fora \\ (UFJF) \\ Caixa Postal 20.010 - 36.036-900 - Juiz de Fora - MG - Brazil \\ \{eduardo.barrere, marlucevitor, miguel.alvim\}@ice.ufjf.br
}

\begin{abstract}
This article highlights the main element of gamification present in Moodle, the ranking. The current version of the ranking, available in the platform plugin repository, presents several limitations verified by means of a case study. From these limitations a new ranking has been developed, optimizing the configuration possibilities and allowing rankings to be defined for specific sessions of a course. As a proof of concept, the ranking was used in a new case study, proving the improvements proposed in this type of gamified element.
\end{abstract}

Resumo. Este artigo destaca o principal elemento de gamificação presente no Moodle, o ranking. A versão atual do ranking, disponivel no repositório de plugins da plataforma, apresenta diversas limitações verificadas por meio de um estudo de caso. A partir dessas limitações foi desenvolvido um novo ranking, otimizando as possibilidades de configuração e permitindo que sejam definidos rankings para sessões específicas de um curso. Como prova de conceito, o ranking foi utilizado em um novo estudo de caso, comprovando as melhorias propostas nesse tipo de elemento gamificado.

\section{Introdução}

Os processos educacionais tradicionais estão sendo muito questionados atualmente. Questões sobre como conseguir um maior engajamento dos alunos da chamada "Geração Y" nas aulas e consequentemente nas atividades é um grande desafio. Uma das formas de "chamar mais a atenção" dos alunos e tornar a dinâmica mais atrativa é a gamificação.

A gamificação utiliza técnicas características de jogos em geral (videogames, jogos de tabuleiros, jogos de cartas, entre outros) em situações do mundo real, sendo aplicada em vários campos de atividades, como produtos comerciais e, no caso desta pesquisa, na área de Educação a Distância (EAD) [Mattar 2016]. A gamificação faz uso de elementos de jogos (mecânica, estratégia, pensamentos e estética) fora do contexto dos jogos, com a finalidade de motivar os indivíduos à ação, auxiliar na solução de problemas e promover aprendizagens [Kapp 2012]. Quando aplicada à educação, a gamificação pode ser definida como a utilização das estratégias características de design de jogos para tornar o processo de aprendizagem mais atrativo, adaptando comportamentos naturais do ser humano como a competitividade, socialização, busca por recompensas e o prazer da superação para atividades educacionais [Fardo 2013]. 
Ao considerar a aplicação da gamificação na educação a distância, as plataformas mais populares para EAD se tornam o caminho mais natural, entre elas se destaca o Moodle como sendo a plataforma mais utilizada na atualidade. Neste cenário, a criação de elementos de gamificação, na forma de um plugin ou módulo, que incentivem a permanência dos estudantes no ambiente virtual de aprendizagem, se faz importante.

Atualmente, existem no ambiente Moodle algumas opções para os professores e administradores gamificarem as atividades desenvolvidas no ambiente de aprendizagem para os alunos. Dentre essas opções se destaca a classificação dos alunos com base numa pontuação e demostrada através de um quadro de pontuações, o chamado ranking. Este elemento apresenta uma série de problemas e limitações. Tais características foram verificadas ao utilizarmos o ranking em um curso massivo para a formação de professores. Visando propor um ranking mais adequado às características do Moodle e ao seu potencial de gamificação, desenvolvemos um novo ranking, com maior flexibilidade de configuração e tentando resolver alguns problemas encontrados anteriormente. Para validar o novo elemento de gamificação, o mesmo foi utilizado em um novo curso massivo para formação de professores de matemática.

O presente artigo apresenta todo este processo e também potenciais ações futuras para aprimorar ainda mais os mecanismos de gamificação na plataforma Moodle.

\section{Gamificação}

As primeiras menções à gamificação são datadas em meados de 2008, como um termo usado pela indústria de mídia digital, com adoção em larga escala a partir do ano de 2010[ Deterding 2011]. O termo se origina da palavra inglesa game (em português, jogo) unida do prefixo "-ation" (em português, "-ação"), gerando o significado de aplicação de games em algo. Atualmente, o termo é ligado à utilização de características envolvidas no design de jogos (eletrônicos, de tabuleiro e outros tipos) em atividades não voltadas, necessariamente, à recreação. $O$ intuito disto é utilizar o potencial de motivação e cativação geralmente presentes em jogos, para incentivar as pessoas envolvidas nestas demais atividades.

A utilização de jogos em outros contextos não lúdicos não é uma iniciativa nova. Em fato, jogos originalmente são uma maneira de seus jogadores treinarem para situações reais em ambientes controlados e simplificados, onde resultados positivos geram recompensas, que por sua vez, levam o jogador a dar maior consideração e peso a ações positivas em situações reais[Koster 2013]. Tentativas de utilizar estas ideias de maneira mais focada a problemas modernos já são feitas à várias décadas e em diferentes níveis de nossa sociedade. Um bom exemplo disto está na área de educação, onde a criação dos ditos jogos educacionais [Koster 2013][Ivetic 2012] já é algo comum desde os anos 70, tendo como um bom exemplo, o jogo "Oregon Trail", originalmente criado em 1971 nos EUA, com o intuito de oferecer aos alunos de escola primária uma visão da vida e desafios dos emigrantes que trilharam a dita "Trilha do Oregon" para colonizarem as terras do atual estado do Oregon - EUA. Com isso em mente, temos que a utilização de jogos, logo Gamificação, nada mais é do que a uma relativamente nova abordagem [Deterding 2011] desta tentativa de unir jogos a processos modernos de trabalho e educação. 
A gamificação vem encontrando aplicações em ambientes digitais [Deterding 2011], onde a maior liberdade de tratamento e armazenamento de dados, assim como acessibilidade através da internet ou diversos tipos de equipamentos, permitem a criação de atividades mais ricas, dinâmicas e acessíveis para seus usuários [Ibáñez 2015].

No âmbito educacional, várias iniciativas acadêmicas de estudo e avanço destas técnicas estão surgindo [Gené 2014][Ibáñez 2015][Kiryakova 2014]. Um exemplo a ser citado é o trabalho de Gené (2014), no qual são utilizadas técnicas de aplicação da gamificação no Moodle para auxiliar na retenção de alunos inscritos em MOOCs (Cursos Online oferecidos para um grande número de estudantes, geralmente ministrados completa ou parcialmente a distância). Temos a criação de cursos reais nos quais técnicas de incentivo oriundas da gamificação (como a bonificação de estudantes via medalhas digitais, desafios semanais e quadros de pontuações) geraram resultados positivos no aumento do engajamento de diversos alunos no conteúdo apresentado [Ibáñez 2015].

Analisando estes estudos, é possível verificar que todos apontam para um aumento na capacidade e interesse de aprendizagem por parte dos alunos envolvidos em cursos que utilizam estas técnicas [Ibáñez 2015][Kiryakova 2014][Barata 2013], com valores quantitativos de aprimoramento chegando a 40\% [Kiryakova 2014]. Também é visto um aumento do interesse de alunos no conteúdo das disciplinas, mesmo após eles já terem sido aprovados nas mesmas [Ibáñez 2015]. Esses resultados demonstram o potencial da criação e aplicação de ferramentas de gamificação no aprimoramento da qualidade de ensino de alunos de cursos superiores [Ibáñez 2015][Barata 2013], apontando para a importância de estudos e aplicações desta nova área.

\section{Gamificação no Moodle}

Existem diversos elementos/componentes que podem ser utilizados para a gamificação em ambientes virtuais de aprendizagem. Conforme abordado por Lister (2015), os elementos de gamificação comumente implementados incluem pontos, quadros de classificação, emblemas e níveis, comumente chamada de PBL triad (Ponits, Badges and Leaderboards). A Figura 1 apresenta a quantidade de trabalhos pesquisados por Lister (2015) que utilizam cada tipo de elemento, mas O'Donnell et al. (2013) destacam que emblemas (badges), barras de progresso e quadros de classificação (ranking) possuem o maior potencial de eficácia dentro de um ambiente educacional.

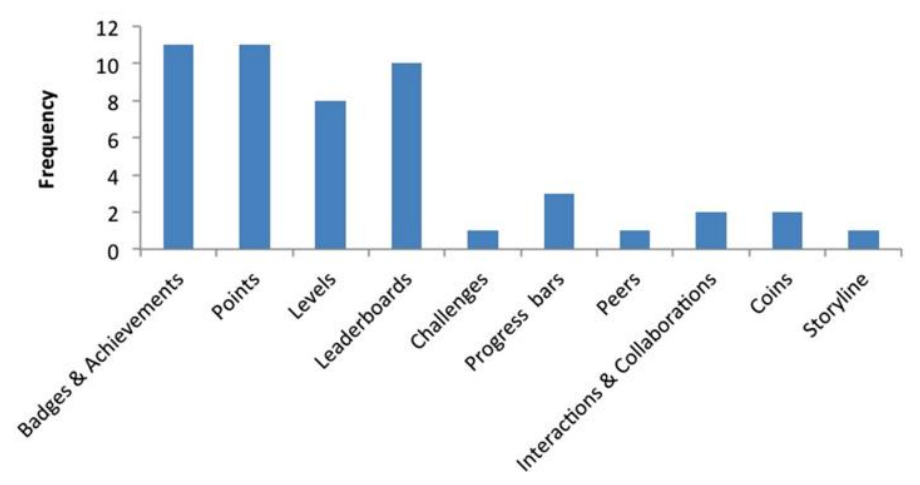

Figura 1. Frequência dos elementos de Gamificação [Lister 2015] 
VI Congresso Brasileiro de Informática na Educação (CBIE 2017)

Anais do XXVIII Simpósio Brasileiro de Informática na Educação (SBIE 2017)

Os trabalhos analisados por Lister (2015) apontam para aspectos interessantes sobre alguns desses elementos:

- Os pontos não podem ser utilizados de forma isolada, ou seja, eles devem estar atrelados a outros elementos de gamificação ou bonificação, como, por exemplo, os quadros de pontuação (ranking). O maior destaque desse elemento é o feedback instantâneo para o aluno.

- Os emblemas incentivam o envolvimento dos alunos, mas um mesmo emblema pode afetar de forma diferente a motivação dos alunos. Lister (2015) apud Abramovich et al. (2013) destaca a diferença nos padrões de aquisição de emblemas para alunos com diferentes níveis de conhecimento prévio. Os alunos de baixo desempenho foram motivados por emblemas concedidos para participação, enquanto os estudantes de alto desempenho foram motivados por emblemas concedidos para aquisição de habilidades.

- O quadro de classificação (ranking) é um elemento de jogo comumente usado. Dependendo do tipo de jogador (socializadores, exploradores, conquistadores ou lutadores [Bartle 1996]; ou outras classificações), o ranking oferece motivação, enquanto outros estudantes não gostam deste elemento de competição. Para alunos competitivos, o quadro de classificação fornece feedback instantâneo e permite que os alunos se esforcem continuamente para melhorar seu lugar/posição no ranking.

Quando o ambiente virtual de aprendizagem é o Moodle, dentre os elementos básicos para a gamificação da plataforma destacam-se:

- Badges (emblemas): Conjuntos de distintivos, medalhas ou troféus. O objetivo é premiar o aluno por ter completado uma tarefa.

- Progress Bar (barra de progresso): Ferramenta de gerenciamento da progressão do aluno no curso.

- Level Up! (nível): Ferramenta que, de acordo com o desempenho dos alunos nas atividades, faz com que o aluno "suba" de nível.

- Ranking Block (quadro de classificação): Plugin de classificação dos participantes de acordo com a nota obtida nas atividades realizadas no Moodle.

Diversos autores apresentam outros elementos de gamificação para a plataforma Moodle, mas acabam não disponibilizando a solução desenvolvida para a comunidade (https://moodle.org/plugins/). Como exemplo vale citar o GameLearning [Roque 2013], um bloco integrado ao ambiente Moodle que agrega técnicas de gamificação às atividades de ensino propostas no ambiente, permitindo gerar um questionário totalmente gamificado.

Neste cenário tomamos como base os elementos disponibilizados através do repositório de plugins do Moodle. Dentre eles, o Ranking Block e o Badges são os que mais se destacam na pesquisa realizada por Lister (2015) e também os que mais se aproximam de elementos de jogos com grande atratividade nos dias atuais. Os badges são bem eficazes e simples, a cada atividade/ação realizada pode ser associada um emblema, que o aluno receberá ao concluí-la. Já o ranking block do Moodle é mais limitado e apresenta alguns problemas discutidos na próxima seção. 
VI Congresso Brasileiro de Informática na Educação (CBIE 2017)

Anais do XXVIII Simpósio Brasileiro de Informática na Educação (SBIE 2017)

\section{Gamificação em um MOOC}

Durante o desenvolvimento de uma dissertação na área de Educação Matemática, foi definida como estratégia a criação de um MOOC para a formação de professores, com foco no uso de tecnologias para o ensino de matemática. O MOOC foi ofertado em 2016 para um grupo inicial de 761 participantes de todas as partes do Brasil. Visando aumentar a retenção no curso, foram utilizados como elementos de gamificação os emblemas e o ranking. Aproximadamente $30 \%$ dos participantes concluíram o curso, o que pode ser considerado uma taxa de conclusão aceitável para cursos dessa natureza.

Os emblemas foram descritos no mapa de atividades do curso e eram "entregues" após o acesso de materiais ou conclusão de atividades, sendo uma das atribuições para o recebimento do certificado. A utilização de emblemas mostrou-se extremamente útil, tanto para a motivação dos alunos, quanto como um facilitador para o controle sobre as atividades concluídas por cada participante. Conforme relato de alunos, através dos emblemas já recebidos e dos emblemas a receber, era possível realizar o gerenciamento sua participação no curso. Alguns relatos destacaram o emblema como um bom mecanismo de retorno (feedback) pelo esforço realizado e, quando um emblema não era emitido por alguma falha no processamento do Moodle, imediatamente o aluno reportava aos professores o problema.

O Ranking foi configurado para pontuar o fórum e demais atividades avaliativas utilizadas no curso (questionários e avaliação por pares). Devido à falta de documentação do plugin, mesmo realizando as configurações, foi inviável entender a real forma de atribuição de pontuação utilizada. Por exemplo, ao atribuir dois pontos a um questionário, o aluno receberia pontos gerados pela conclusão do questionário (dois pontos) mais os pontos das questões certas. Esta situação somente foi esclarecida após estudarmos o código fonte do plugin. Sendo assim, o ranking trouxe um enorme incômodo aos participantes, por não entenderem o seu funcionamento. Um aspecto positivo sobre a utilização do ranking foi verificar a preocupação dos participantes quanto à forma de avaliação, pois foram diversas as perguntas sobre a validade do ranking para a aquisição do certificado, mesmo que este aspecto hora alguma tenha sido abordado, o que evidencia que esse elemento de gamificação causou impacto sobre a forma como os participantes interagiam com o curso.

Após o término do curso e todos os problemas gerados pelo ranking block, ficamos motivados a desenvolver/otimizar a solução proposta atualmente no Moodle para este tipo, tão importante, de elemento de gamificação. Neste cenário, como ação inicial, foram listadas as limitações presentes no ranking atualmente disponível no repositório de plugins do Moodle:

1) Falta de documentação que esclareça como as pontuações são calculadas.

2) Não permitir a atribuição de pontos para qualquer atividade/ação do Moodle.

3) O grau de configuração é muito baixo, gerando muita insatisfação por parte dos alunos, acostumados a grande dinâmica existente nos jogos, pois somente é possível atribuir pontos para a atividade e não para as etapas da atividade. 
4) Os rankings existentes são: geral, semana e mês; sendo que os dois últimos normalmente não representam sessões ou partes delimitadas do curso, gerando grande confusão por parte dos alunos.

5) A simples postagem de um "ok" no fórum gera a mesma pontuação no ranking que qualquer outro tipo de postagem que abra caminho para uma longa discussão. Essa limitação foi muito questionada pelos alunos que acompanham a sua pontuação e a de outros colegas para ver quem ficava na frente no quadro de pontuações. Eles rapidamente perceberam que a qualidade das postagens era irrelevante para o ranking. Esse aspecto é reforçado pela pesquisa de Meyer (2008), que examinou o impacto dos pontos sobre a qualidade das postagens em um fórum de discussão, seguindo o modelo atual de ranking do Moodle.

\section{Novo Ranking}

Considerando o cenário apresentado na seção anterior, algumas possibilidades se apresentaram e serviram de motivação para a criação de um novo ranking. As premissas para esse novo ranking foram:

- No ranking block existe uma limitação com relação à pontuação atribuída a cada atividade, se o professor/administrador atribuir uma nota abaixo de 2 , o valor atribuído a cada atividade ou recurso não altera, pois a nota mínima permitida é 2 . Portanto, o ranking proposto deve permitir qualquer pontuação $(0,1,2 \ldots$.$) .$

- Aumentar o grau de configuração das pontuações atribuídas (granularidade), como por exemplo, permitir a pontuação de todas as ações no fórum (postar tópicos, responder, número de respostas etc.).

- Possibilitar a atribuição de pontuação para atividades, fóruns e também recursos (arquivos, mídias etc.).

- Permitir a criação de diversos rankings para o mesmo curso. Além do ranking geral do curso, ser possível criar ranking por sessões/tópicos e não mais por tempo.

Um das características da versão atual do ranking block, desenvolvida por Willian Mano, é a forma de atribuição dos pontos. A solução de Willian é baseada em eventos, o que só permite ao plugin adicionar pontos para atividades com critérios de conclusão, com isso os pontos são adicionados em tempo real. Então, dependendo de como a tarefa/recurso é configurado, o aluno recebe a pontuação após terminar a atividade ou acessar o recurso, dependendo exclusivamente da configuração da atividade/recurso. Este aspecto é um limitador importante para a alteração das configurações do ranking após o início do curso, pois pontuações atribuídas não podem ser recalculadas por estarem associadas a eventos passados que as geraram. A proposta aqui apresentada segue a mesma linha para atribuir os pontos, a ativação pelo evento de conclusão da atividade (automático ou acionado pelo aluno ou professor/tutor).

O novo ranking foi desenvolvido para a versão 3.2.1 do Moodle. A Figura 2 apresenta um exemplo da tela de configuração. Além de atender às premissas descritas no início desta seção, ele apresenta como características:

- Não utilizar as notas atribuídas para a atividade como pontos no ranking. Ele apenas contabiliza a pontuação configurada pelo administrador/professor do curso. 
VI Congresso Brasileiro de Informática na Educação (CBIE 2017)

Anais do XXVIII Simpósio Brasileiro de Informática na Educação (SBIE 2017)

O objetivo foi possibilitar ao aluno entender melhor a sua pontuação e de seus colegas, visando tornar mais clara a forma de competição.

- Possibilitar a existência de rankings por sessão do Moodle, em vez de utilizar o ranking por semana ou mês, pois enquanto as atividades e a sessão estiverem ativas, o aluno poderá ganhar pontos no ranking " $X$ ", independentemente da semana ou mês atual. Tal procedimento faz com que diminua os dilemas em relação à classificação no quadro de pontuação da sessão. Como contraexemplo no ranking por semana, o aluno pode fazer as atividades dos módulos I e II (desde que as atividades estejam ativas) e ficar em primeiro colocado, naquela semana.

- No fórum, além da pontuação geral por participação, é permitido pontuar abertura de discussão, réplicas e tréplicas. Tal fator é importante para incentivar o debate. Para que o fórum pontue corretamente, é necessário que o tutor/professor avalie as postagens ou que o aluno fique com a ação de concluir a atividade, gerando assim o evento que aciona a contagem da pontuação.

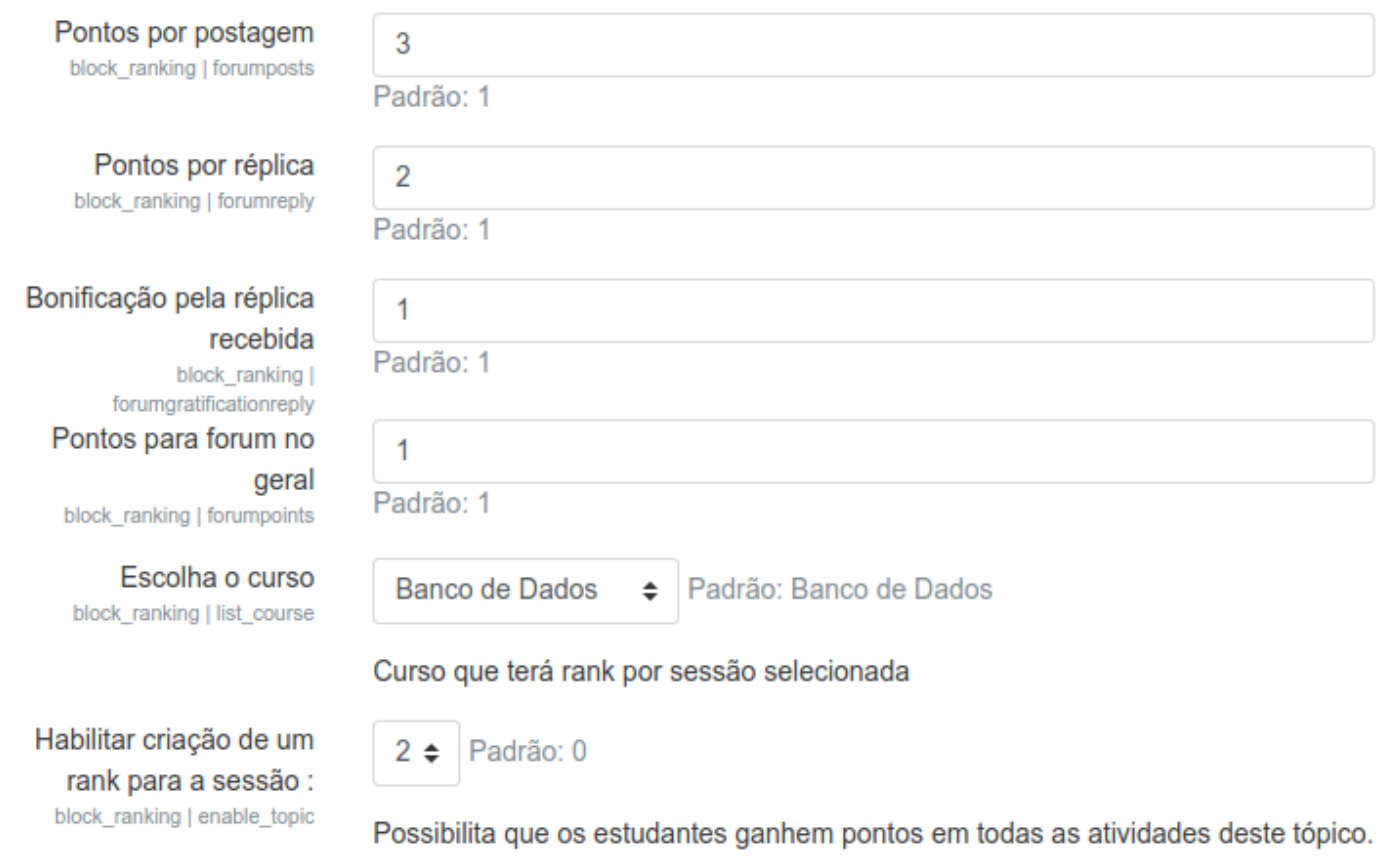

Figura 2. Configuração das funcionalidades específicas do novo ranking

\section{Estudo de caso}

Dada a necessidade de utilização de gamificação em outro curso massivo para a área de Educação Matemática, foi definida a utilização do novo ranking em substituição do ranking block. Tal possibilidade é reforçada pelo fato dos participantes deste novo MOOC terem um perfil similar ao do MOOC anterior, professores de matemática em busca de formação continuada. Esta similaridade foi confirmada após a execução do mesmo, ao verificar que a divisão dos participantes por faixa etária, formação e conhecimento tecnológico, ser muito similar para ambos os cursos. O MOOC foi ofertado no início de 2017, com a participação de 511 professores, e os participantes foram divididos em 5 grupos com a finalidade de melhor gestão da dinâmica do curso. 
VI Congresso Brasileiro de Informática na Educação (CBIE 2017)

Anais do XXVIII Simpósio Brasileiro de Informática na Educação (SBIE 2017)

Para facilitar a contabilização dos pontos, todas as ações gamificadas (recursos, enquetes, questionários, fóruns e avaliação por pares) tiveram como atribuição um ponto. Vale ressaltar que, para o fórum, o aluno recebe um ponto pela sua participação e por cada postagem feita como resposta a sua (réplicas e tréplicas), desde que não tenham sido feitas por ele mesmo. Foram habilitados cinco rankings, correspondentes às cinco sessões do curso (Módulos de I a V) e o ranking geral.

A Figura 3 ilustra um dos gráficos de gestão disponível para o professor/administrador do curso, através do qual é apresentada a pontuação média de cada grupo. Além deste gráfico, é possível verificar a variação de pontos obtida pelos participantes do grupo em cada dia da semana, vide Figura 4, demonstrando quais os dias da semana os alunos daquele grupo mais concluem as atividades da sessão vigente.

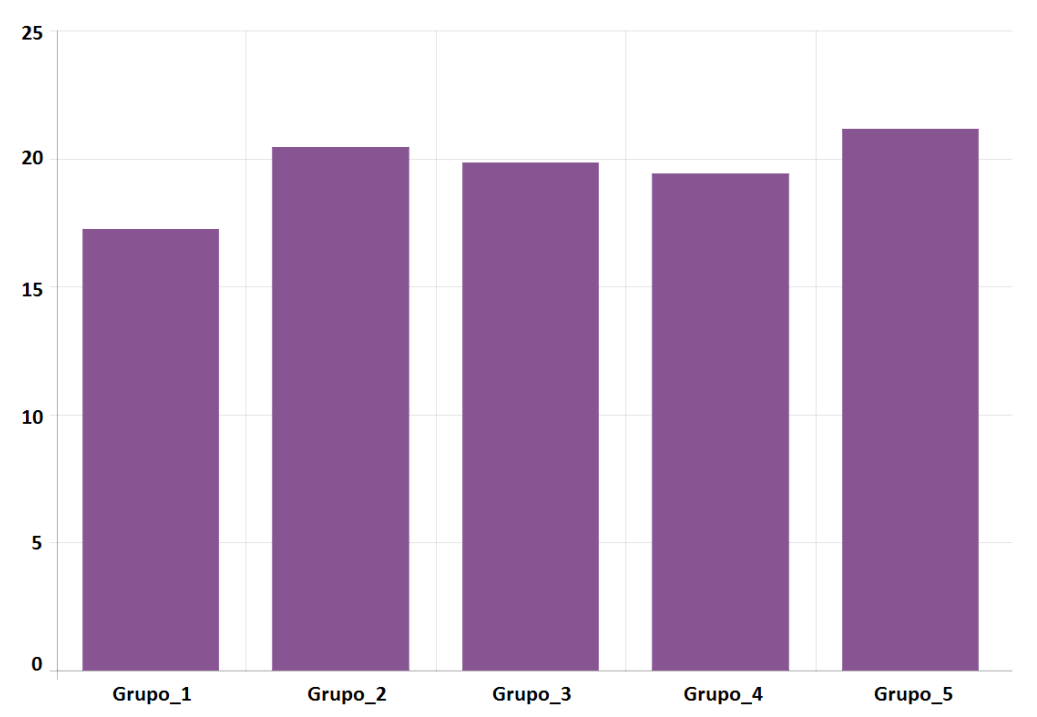

Figura 3. Gráfico da média de pontos por grupo

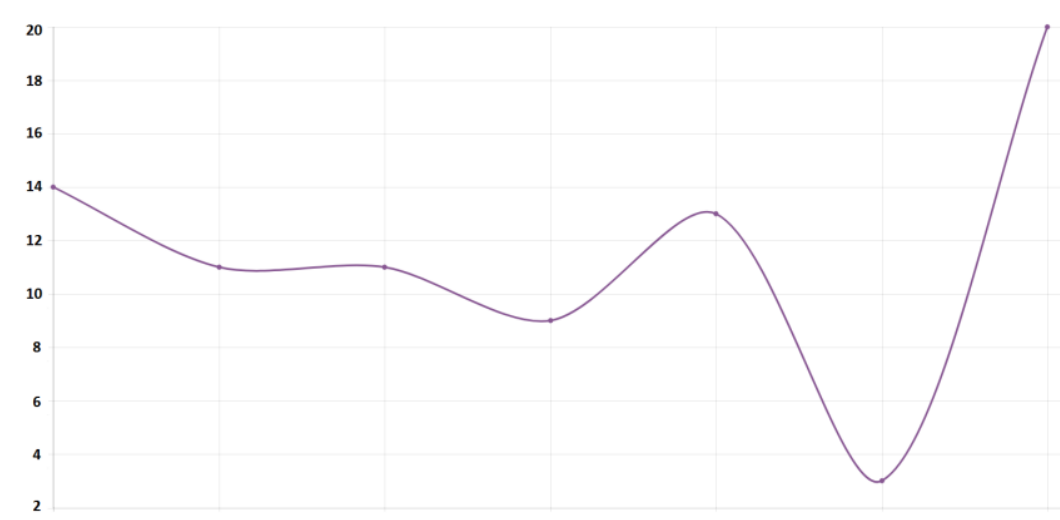

Figura 4. Gráfico da evolução dos pontos atribuídos a grupo em uma semana

De forma geral foi possível concluir que houve uma diminuição em relação à quantidade de reclamações relacionadas ao ranking, principalmente forma de pontuação e delimitação dos rankings e não entendimento dos pontos atribuídos, tendo diminuído de $15 \%$ para aproximadamente $1 \%$ da turma. Os alunos entenderam melhor a dinâmica da pontuação e dos rankings, fato que aumentou a satisfação para com o curso. 
Algumas configurações inadequadas dificultaram a atribuição dos pontos, como o fato do aluno poder marcar o fórum como realizado e continuar a postar ou receber postagens relativas às suas colocações. Tal situação fez com que o evento para atribuir a pontuação tivesse ocorrido antes mesmo das postagens, ou seja, os pontos que deveriam ser atribuídos não foram. Para resolver tal problema, é necessário configurar a conclusão do fórum para o final da sessão e informar aos alunos que postagens posteriores à conclusão temporal da sessão não serão contabilizadas.

\section{Conclusões}

Tanto na literatura, quanto nos estudos de caso desenvolvidos é possível perceber que a utilização de emblemas e rankings compõe uma forma prática e eficiente de gamificar uma plataforma de EAD. No caso específico do ranking, os participantes de um curso a distância têm no quadro de pontuação uma forma de comparar seu desempenho em relação aos seus colegas e se motivarem a participar de atividades e acessar diversos conteúdos. Apesar de todo esse cenário a favor, a implementação atual de ranking disponível no repositório de plugins do Moodle apresenta uma série de limitações, podendo destacar a periodicidade dos rankings e a forma confusa de pontuação.

O presente trabalho analisou essas questões e apresentou um novo ranking que resolve os problemas acima destacados e outros mais. No segundo estudo de caso realizado, as reclamações e dúvidas relacionadas ao ranking diminuíram bastante em relação ao primeiro estudo de caso, sendo que a maioria dos questionamentos pode ser resolvida com um esclarecimento sobre como os pontos são atribuídos e em qual ranking são computados. Esta ação não foi realizada durante o curso para não gerar alterações significativas no comportamento dos usuários, mas ficou registrado como ação a ser realizada para os próximos cursos. Outro fator positivo do novo ranking foi a participação dos alunos nos fóruns, incentivada pela forma de pontuação relacionada às réplicas e tréplicas, mas falta a avaliação qualitativa dessas interações, que será feita como trabalho futuro.

Para evoluir o novo ranking e poder disponibilizá-lo no repositório de plugins do Moodle, se faz necessário ampliar a quantidade e parametrização dos gráficos, permitir que o professor/tutor possam limpar os registros de pontuação de um determinado curso, por exemplo, enquanto estiver em fase de testes. Também é necessário criar toda a documentação do plugin, tanto relativa à codificação quanto a parte de configuração e uso. Por último, permitir que os emblemas possam contar pontos no ranking, ou seja, deve existir uma opção na configuração para tal.

\section{Referências}

Abramovich, S., Schunn, C., and Higashi, R. (2013) "Are badges useful in education?: It depends upon the type of badge and expertise of learner", Educational Technology Research and Development, 61(2), p. 17-232.

Barata, G., Gama, S., Jorge, J. and Gonçalves, D. (2013) "Improving Participation and Learning with Gamification", Gamification '13 Proceedings of the First International Conference on Gameful Design, Research, and Applications, p. 10-17. 
VI Congresso Brasileiro de Informática na Educação (CBIE 2017)

Anais do XXVIII Simpósio Brasileiro de Informática na Educação (SBIE 2017)

Bartle, R. (1996) "Hearts, Clubs, Diamonds and Spades: Players who suit MUDs", Journal of MUD research, p. 1-19.

Deterding, S. et al. (2011) "From game design elements to gamefulness: defining gamification", ACM. Proceedings of the 15th international academic MindTrek conference: Envisioning future media environments, p. 9-15.

Falcão, A.P., Leite, M.D. and Tenório, M.M. (2014) "Ferramenta de apoio ao ensino presencial utilizando gamificação e design de jogos" XXV Simpósio Brasileiro de Informática na Educação (SBIE 2014), p. 526-533.

Fardo, M.L. (2013) “A Gamificação Aplicada em Ambientes de Aprendizagem", RENOTE - Revista Novas Tecnologias na Educação, volume 11, número 1.

Gené, O. B., Nuñez, M. M. and Blanco, Á. F. (2014) "Gamification in mooc: challenges, opportunities and proposals for advancing mooc model", ACM. Proceedings of the Second International Conference on Technological Ecosystems for Enhancing Multiculturality, p. 215-220.

Ibáñez, M. B., Di-Serio, A. and Delgado-Kloos, C. (2014) "Gamification for engaging computer science students in learning activities: A case study", IEEE Transactions on Learning Technologies, IEEE, v. 7, n. 3, p. 291-301.

Ivetic, D. et al. (2012) "Gamifying education: A proposed taxonomy of satisfaction metrics", Conference proceedings of eLearning and Software for Education (eLSE), p. 345-350.

Kapp, K.M. (2012). The Gamification of Learning and Instruction: Game-based Methods and Strategies for Training and Education, Pfeiffer.

Kiryakova, G., Angelova, N. and Yordanova, L. (2014) "Gamification in education", Proceedings of 9th International Balkan Education and Science Conference.

Koster, R. (2013). A Theory of Fun for Game Design, 2nd Edition. O’Reilly Media Inc, 2013. ISBN 978-1-449-36321-5

Lister, M. C. (2015) "Gamification: The effect on student motivation and performance at the post-secondary level", Issues and Trends in Educational Technology Volume 3, Number 2, Dec. 2015.

Mattar, J. and Nesteriuk, S. (2016) "Estratégias do design de games que podem ser incorporadas à educação a distância", RIED - Revista Iberoamericana de Educação a Distancia, volume 19, número 2.

Meyer, K. (2008) "Do rewards shape online discussions?", Journal of Interactive Online Learning, 7(2), p. 126-138.

O'Donovan, S., Gain, J., and Marais, P. (2013) "A case study in the gamification of a university-level games development course", Proceedings of the South African Institute for Computer Scientists and Information Technologists Conference, (SAICSIT'13), p. 242-251.

Roque, A.S., Paludo, C. and Geiss, E. (2013) "GameLearning e suas Contribuições ao Ambiente Virtual de Aprendizagem Moodle", Nuevas Ideas en Informática Educativa (TISE 2013). p. 677-680. 